\title{
Fractional-Dose Inactivated Poliovirus Vaccine Immunization Campaign - Telangana State, India, June 2016
}

\author{
Sunil Bahl, MD ${ }^{1}$; Harish Verma, MBBS, DCH${ }^{2}$; Pankaj Bhatnagar, $\mathrm{MD}^{3}$; Pradeep Haldar, MBBS ${ }^{4}$; Asish Satapathy, MBBS $^{3}$; \\ K. N. Arun Kumar, MBBS 3 ; Jennifer Horton, $\mathrm{MD}^{2}$; Concepcion F. Estivariz, MD ${ }^{5}$; Abhijeet Anand, MBBS ${ }^{5}$, Roland Sutter, $\mathrm{MD}^{2}$
}

Wild poliovirus type 2 was declared eradicated in September 2015 (1). In April 2016, India, switched from use of trivalent oral poliovirus vaccine (tOPV; containing types 1,2 , and 3 polio vaccine viruses), to bivalent OPV (bOPV; containing types 1 and 3), as part of a globally synchronized initiative to withdraw Sabin poliovirus type 2 vaccine. Concurrently, inactivated poliovirus vaccine (IPV) was introduced into India's routine immunization program to maintain an immunity base that would mitigate the number of paralytic cases in the event of epidemic transmission of poliovirus type $2(2,3)$. After cessation of use of type 2 Sabin vaccine, any reported isolation of vaccine-derived poliovirus type 2 (VDPV2) would be treated as a public health emergency and might need outbreak response with monovalent type 2 oral vaccine, IPV, or both (4). In response to identification of a VDPV2 isolate from a sewage sample collected in the southern state of Telangana in May 2016, India conducted a mass vaccination campaign in June 2016 using an intradermal fractional dose $(0.1 \mathrm{ml})$ of IPV (fIPV). Because of a global IPV supply shortage, fIPV, which uses one fifth of regular intramuscular (IM) dose administered intradermally, has been recommended as a response strategy for VDPV2 (5). Clinical trials have demonstrated that fIPV is highly immunogenic $(6,7)$. During the 6-day campaign, 311,064 children aged 6 weeks-3 years were vaccinated, achieving an estimated coverage of $94 \%$. With appropriate preparation, an emergency fIPV response can be promptly and successfully implemented. Lessons learned from this campaign can be applied to successful implementation of future outbreak responses using fIPV.

On June 7, 2016, a VDPV2 isolate with 10 nucleotide changes from the corresponding OPV strain was reported in an environmental surveillance sample collected from a sewage site on May 16, 2016. The sample was collected from the Amberpet sewage treatment plant, which receives sewage from parts of the Hyderabad and Rangareddy districts of Telangana state (Figure). No cases of acute flaccid paralysis (AFP) caused by poliovirus were reported and an active search of medical records in health care facilities identified no unreported AFP cases in Hyderabad and Rangareddy districts in the preceding 6 months. The last reported case of wild poliovirus case in Telangana state occurred in 2007, and no VDPVs had been reported from any sampling site since initiation of environmental sampling in Hyderabad in April 2016.
Following the outbreak response protocol for a VDPV2 event (4), which calls for an immediate vaccination response and after a joint national (Governments of India and Telangana State) and international (Global Polio Eradication Initiative [GPEI]) review, a decision was made to conduct a campaign using fIPV. The target group for the fIPV campaign was children aged 6 weeks -3 years. The campaign was limited to areas from which sewage drains to the Amberpet sewage treatment plant and that were considered to be at high risk for a potential circulating VDPV (cVDPV) outbreak, based on coverage of routine immunization and quality of earlier polio vaccination campaigns (i.e., Hyderabad city/district), and areas adjoining slum and migrant populations at high risk in the Rangareddy district.

In contrast to the house-to-house approach used for OPV campaigns, during which OPV is administered to targeted children directly at their home or other points where they are encountered (e.g., bus stops or public markets), the fIPV campaign was implemented using a "fixed site" approach, in which parents and caretakers bring children to a convenient neighborhood location for vaccination. The campaign was implemented June 20-25, 2016, within the recommended maximum 14-day interval to conduct a response after the initial confirmation of VDPV (4).

\section{Campaign Planning and Implementation}

Twenty-nine surveillance medical officers from World Health Organization-India's National Polio Surveillance Project were deployed to support the development of campaign microplans and to conduct precampaign training and campaign monitoring. Existing microplans* developed for previous OPV campaigns were adapted for the fIPV campaign. A rapid house-to-house survey was conducted to enumerate all eligible children and to inform families about the campaign. The target population was estimated to be 291,305 .

A total of 5,373 immunization sessions were organized during 6 days (Table 1); the number of daily sessions ranged from 719 to 1,227 . A total of 1,038 vaccinators supported implementation of the campaign, with 638 vaccinators mobilized from neighboring districts. At least one four-member team that included one vaccinator (an auxiliary nurse midwife),

\footnotetext{
*http://www.who.int/immunization/sage/9_Final_RED_280909.pdf.
} 


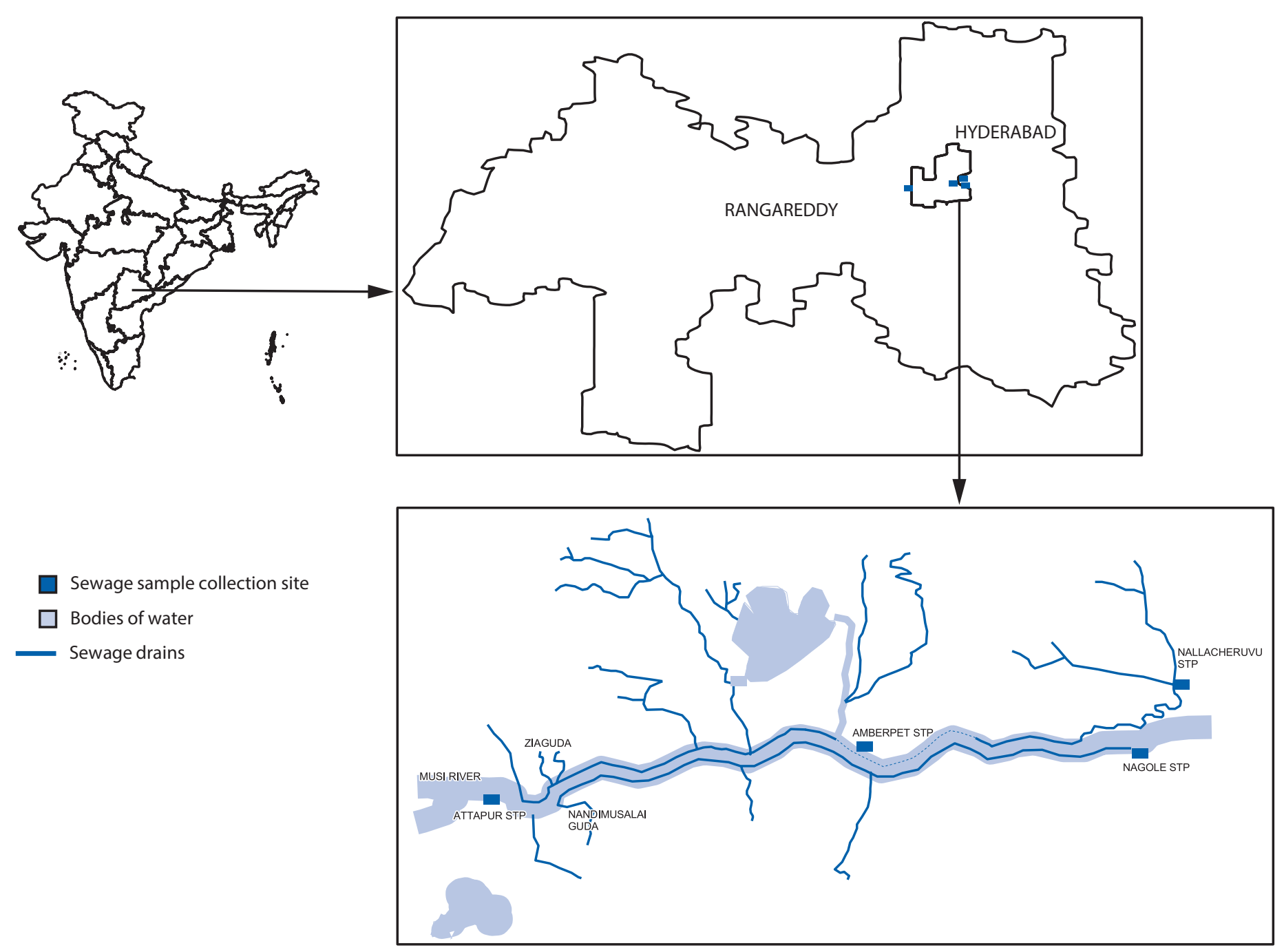

Abbreviation: STP = sewage treatment plant.

two community mobilizers, and one volunteer managed each session. Auxiliary nurse midwives administer all injectable vaccines during routine immunization sessions. A 1-day training session was organized to instruct all vaccination staff members supporting the fIPV campaign. Social mobilization for the campaign was conducted through print and electronic media, posters, invitation slips to parents of eligible children indicating day and place of immunization sessions, banners, microphone announcements, and community mobilizers.

The IPV vials used in the campaign were 10-IM-dose vials ( $5 \mathrm{ml}$ per vial) manufactured by Shantha Biotech (Hyderabad, India) with $0.1 \mathrm{ml}$ withdrawn for each fIPV vaccination. Therefore, each 10-IM-dose vial could potentially vaccinate 50 children with fIPV. The multi-dose vial policy permitted use of open IPV vials for up to 28 days from the date of first use (8); partially used opened vials returned at the end of each campaign day were the first priority for use during the next day. A 0.1-ml dose of fIPV was administered intradermally on the lateral aspect of the right upper arm using an autodisabled needle and syringe (with a 0.1-ml mark). An autodisabled needle/syringe is a "fixed system," in which the needle cannot be removed from the syringe; this system reduces vaccine wastage from the syringe. After vaccination, the nail of the left fifth finger of each vaccine recipient was marked with an indelible marker pen. Parents and caregivers were asked to report any adverse events occurring within a week of receiving the vaccine, including illness, hospitalizations, or death. 


\section{Campaign Monitoring}

At least one campaign monitor was assigned to each of the 25 blocks/administrative divisions in the districts of Hyderabad and Rangareddy. Areas selected for monitoring were known locations of residence of disenfranchised, mobile, or migrant populations and other groups for which lower than average routine immunization coverage had been reported. A total of $958(18 \%)$ vaccination sessions were observed during the 6-day campaign (Table 2). All monitored team sessions were organized as planned, and $96 \%$ of monitored teams had the vaccinator that was listed in the microplan. Among monitored teams, $97 \%$ had adequate supplies to conduct vaccination sessions. Because of high vaccine demand, especially on the first 2 days of the campaign, $6 \%$ of monitored teams reported a shortage of IPV vials at some time during the session. No frozen IPV vials were reported to have been observed; IPV is freezesensitive, and any vials that are suspected to have been frozen must be discarded (9). Also, on the basis of their observance of vaccine vial monitors (heat-sensitive labels placed on vaccine vials that register cumulative heat exposure), monitors reported that no IPV vials reached the discard point, which implied an overall appropriate maintenance of the cold chain. On the first day of the campaign, a median of $48 \mathrm{fIPV}$ doses (range: 41-50) were extracted from each IPV vial. Monitors noted that no vaccine leakage from the vial caps occurred during monitored sessions. In $93 \%$ of observed children, a bleb, indicative of intradermal delivery of fIPV, was observed immediately after vaccination. A median of 73 children (range: 10-148) were vaccinated per session per day during the campaign.

\section{Postcampaign Evaluation and Coverage}

A total of 311,064 children were reported to have been vaccinated during the campaign, representing $107 \%$ of the initially estimated target of 291,305 children (Table 1 ). The reported coverage in Hyderabad was $87 \%$ of the estimated target, and in the Rangareddy district, almost twice the number of initially estimated children (185\%) received the vaccine. The high reported coverage in Rangareddy was attributed to a large number of children from nontargeted areas that were adjacent to targeted areas who received vaccine during the campaign. After the campaign, a postcampaign assessment was conducted by 46 monitors to check for the likely number of missed children in a given location. Monitors prioritized areas that were known locations of residence of disenfranchised, migrant, or mobile populations. A total of 2,821 children were randomly checked (through finger marking) by monitors as part of a postcoverage monitoring survey, and $94 \%$ of assessed children overall were found to have received fIPV during the campaign (Table 2). The main reasons for nonvaccination included the
TABLE 1. Numbers of fractional dose of inactivated poliovirus vaccine $(\mathrm{fIPV})^{*}$ vaccination sessions and children (aged 6 weeks -3 years) targeted and vaccinated, and median number of children vaccinated per day in a vaccination session, by district - Telangana, India, June 20-25, 2016

\begin{tabular}{|c|c|c|c|c|}
\hline \multirow[b]{2}{*}{ District } & \multirow{2}{*}{$\begin{array}{l}\text { No. } \\
\text { vaccination } \\
\text { sessions } \\
\text { conducted }\end{array}$} & \multicolumn{3}{|c|}{ Children aged 6 weeks- 3 years } \\
\hline & & No. targeted & $\begin{array}{l}\text { No. reported } \\
\text { vaccinated } \\
\text { with fIPV (\%) }\end{array}$ & $\begin{array}{c}\text { Median no. vaccinated } \\
\text { per day } \\
\text { in a session (range) }\end{array}$ \\
\hline Hyderabad & 4,360 & 231,482 & $200,480(87)$ & $68(10-102)$ \\
\hline Rangareddy & 1,013 & 59,823 & $110,584(185)$ & $87(24-148)$ \\
\hline Total & 5,373 & 291,305 & $311,064(107)$ & $73(10-148)$ \\
\hline
\end{tabular}

* Equivalent to one fifth of an intramuscular dose.

TABLE 2. Monitoring and evaluation of fractional inactivated poliovirus vaccine campaign sessions and children's vaccination status, by district - Telangana, India, June 2016

\begin{tabular}{|c|c|c|c|c|c|}
\hline \multirow[b]{2}{*}{ District } & \multicolumn{4}{|c|}{ Campaign monitoring } & \multirow{2}{*}{$\begin{array}{c}\text { Post-campaign } \\
\text { evaluation } \\
\text { No. children } \\
\text { checked by } \\
\text { monitors for } \\
\text { vaccination } \\
\text { status } \\
\text { (\% vaccinated)* }\end{array}$} \\
\hline & $\begin{array}{c}\text { No. } \\
\text { monitors }\end{array}$ & $\begin{array}{l}\text { No. sessions } \\
\text { monitored (\%) }\end{array}$ & $\begin{array}{l}\text { Sessions with } \\
\text { the vaccinators } \\
\text { listed in } \\
\text { microplan } \\
\text { (\%) }\end{array}$ & $\begin{array}{l}\text { Sessions with } \\
\text { adequate } \\
\text { vaccine/ } \\
\text { syringes } \\
(\%)\end{array}$ & \\
\hline Hyderabad & 30 & $661(15)$ & (95) & (98) & $1,862(96)$ \\
\hline Rangareddy & 16 & $297(29)$ & (98) & (94) & $959(91)$ \\
\hline Total & 46 & 958 (18) & (96) & (97) & $2,821(94)$ \\
\hline
\end{tabular}

* Based on examination of finger marking (after vaccination, the nail of the fifth finger of the left hand of each vaccine recipient was marked with an indelible marker pen).

child was not available on the day of vaccination (29\%), the child was sick (21\%), lack of parental awareness (16\%), fear of injection (2\%), and hesitancy and refusal (6.2\%). Four nonserious adverse events, reported within a week after receipt of fIPV, deemed to be unrelated to vaccination.

\section{Discussion}

Although vaccination campaigns with injectable vaccines have been conducted for other diseases, globally, this was the first campaign to use fIPV, which required vaccinators with experience in administering intradermal injections. Overall, this emergency response to a reported VDPV2 event demonstrates that it is feasible to plan and implement a fIPV campaign within 14 days of the reported event and to achieve high reported coverage. Strong government leadership at the national and state levels, well-coordinated technical and operational support from GPEI partners, clearly defined standard operating procedures for outbreak response, and experience implementing OPV campaigns were critical elements to the success of the fIPV campaign in Telangana state, India.

A number of lessons learned from this experience are likely to aid India and other countries in the successful implementation of future flPV campaigns, as well as emergency campaigns with other injectable vaccines. Meticulous planning to ensure a 


\section{Summary}

What is already known about this topic?

In April 2016, India withdrew Sabin poliovirus type 2 vaccine as part of a globally synchronized initiative that followed the declaration of eradication of wild poliovirus type 2 in September 2015. After the use of Sabin poliovirus type 2 ceased, any report of isolation of vaccine-derived poliovirus type 2 (VDPV2) would be considered a public health emergency and might require an outbreak response vaccination with monovalent type 2 oral polio vaccine or inactivated poliovirus vaccine (IPV). Global IPV supply shortage has limited the number of available doses of IPV. Fractional IPV (fIPV), administered intradermally using one fifth of regular dose, stretches the limited supplies of IPV and has been recommended as a response strategy for VDPV2 outbreaks.

What is added by this report?

In response to a VDPV2 isolation in Telangana, India, a mass vaccination campaign was conducted using fIPV within 14 days of the VDPV2 isolation. A total of 311,064 children were reported to have been vaccinated during the campaign. This was the first mass vaccination campaign to use fIPV, and in a postcoverage monitoring survey, $94 \%$ of assessed children were found to have received fIPV during the campaign.

What are the implications for public health practice?

This emergency response to a reported VDPV2 event demonstrates the feasibility of planning and implementing an fIPV campaign within 14 days of a reported event and of achieving high reported coverage. Strong government leadership at the national and state levels and well-coordinated technical and operational support from Global Polio Eradication Initiative partners, as well as experience in implementing oral poliovirus campaigns and having clearly defined standard operating procedures for outbreak response were critical elements to the success of the fIPV campaign in Telangana, India.

sufficient number of vaccination sites that are located strategically and availability of an adequate number of vaccinators with experience in intradermal administration of vaccines, coupled with rapid refresher training of these vaccinators, assisted in ensuring good injection practices and high coverage as determined by postcampaign monitoring.

The Emergency Operations Center established by the Indian government was responsible for the overall coordination of the emergency response, with strong support from GPEI partners. Communication technologies, such as group messaging, helped ensure rapid communication among all stakeholders. Progress in all sectors of the campaign area was shared in real-time during the preparatory and implementation phases, as were challenges and barriers, to ensure faster solutions to identified problems.

Sharing accurate and timely information is important in developing a positive partnership with the media. Extensive publicity of the campaign through mass media, the perceived threat of the return of polio, and the nonavailability of IPV in the private sector, as well as the private sector's promotion of the campaign, all contributed to high community participation and high coverage.

One unanticipated problem was that the number of children identified during precampaign surveys did not match the number of children who reported to the vaccination sites to receive fIPV, especially in Rangareddy, where only about half the number of children who were actually vaccinated were initially targeted. Therefore, when planning for a time-sensitive outbreak response, resource-intensive precampaign surveys should be avoided. Available resources should be diverted to update existing vaccination microplans and develop communication strategies. Strong mobilization measures using community health workers and volunteers on the days of the campaign were effective in achieving high coverage. This large, emergency campaign with an injectable vaccine required a large number of trained vaccinators to be brought in to the targeted area from other districts; this scenario needs to be anticipated in response plans for future similar campaigns.

The experience in Telangana state, India, demonstrates that operational and logistical challenges to an injectable vaccination campaign can be overcome. Through active government and partner coordination, achievement of high vaccination coverage with intradermal fIPV in an emergency campaign setting is possible.

\footnotetext{
${ }^{1}$ World Health Organization, South-East Asia Regional Office, New Delhi, India; ${ }^{2}$ World Health Organization, Geneva, Switzerland; ${ }^{3}$ National Polio Surveillance Project, World Health Organization, New Delhi, India; ${ }^{4}$ Ministry of Health and Family Welfare, Government of India, New Delhi, India; ${ }^{5}$ Global Immunization Division, Center for Global Health, CDC.
}

Corresponding author: Abhijeet Anand, aanand@cdc.gov, 404-639-1970.

\section{References}

1. Adams A, Salisbury DM. Eradicating polio. Science 2015;350:609. http:// dx.doi.org/10.1126/science.aad7294

2. Global Polio Eradication Initiative. Polio eradication and endgame strategic plan 2013-2018. Geneva, Switzerland: Global Polio Eradication Initiative; 2013. http://www.polioeradication.org/resourcelibrary/ strategyandwork.aspx

3. World Health Organization. Polio vaccines: WHO position paperMarch 2016. Wkly Epidemiol Rec 2016;12:145-68.

4. Global Polio Eradication Initiative. Responding to a poliovirus event and outbreak. Geneva, Switzerland: Global Polio Eradication Initiative; 2016. www.polioeradication.org/Portals/0/Document/Resources/ PolioEradicators/1a.PolioOutbreakGuideline201604part2.pdf

5. World Health Organization; SAGE Polio Working Group. Notes from SAGE Polio Working Group Meeting, March 3, 2016. Geneva, Switzerland: World Health Organization; 2016.

6. Estívariz CF, Jafari H, Sutter RW, et al. Immunogenicity of supplemental doses of poliovirus vaccine for children aged 6-9 months in Moradabad, India: a community-based, randomised controlled trial. Lancet Infect Dis 2012;12:128-35. http://dx.doi.org/10.1016/S1473-3099(11)70190-6

7. Resik S, Tejeda A, Mach O, et al. Immune responses after fractional doses of inactivated poliovirus vaccine using newly developed intradermal jet injectors: a randomized controlled trial in Cuba. Vaccine 2015;33:307-13. http://dx.doi.org/10.1016/j.vaccine.2014.11.025 
8. World Health Organization; United Nations Children's Emergency Fund. Application of WHO multi-dose vial policy for inactivated polio vaccine. Geneva, Switzerland: World Health Organization; 2014. http://www. who.int/immunization/diseases/poliomyelitis/inactivated_polio_vaccine/ MDVP_Nov2014.pdf
9. Global Polio Eradication Initiative. Introduction of inactivated poliovirus vaccine in routine immunizations. Geneva, Switzerland: Global Polio Eradication Initiative; 2014. 